\title{
On the Period Change of the Contact Binary GW Cephei
}

\author{
Chun-Hwey Kim ${ }^{1,2 \dagger}$, Mi Hwa Song ${ }^{1}$, Joh-Na Yoon ${ }^{2}$, Jang Hae Jeong ${ }^{1,2}$, Taek-Soo Jeoung ${ }^{1,2}$, \\ Young-Jae $\mathrm{Kim}^{1,2}$, and Jung Yeb Kim ${ }^{1,2}$
}

${ }^{1}$ Department of Astronomy and Space Science, Chungbuk National University, Cheongju 361-763, Korea

${ }^{2}$ Chungbuk National University Observatory, Chungbuk National University, Cheongju 361-763, Korea

BVR CCD observations of GW Cep were made on 15 nights in November through December 2008 with a 1-m reflector at the Jincheon station of the Chungbuk National University Observatory. Nineteen new times of minimum lights for GW Cep were determined and added to a collection of all other times of minima available to us. These data were then intensively analyzed, by reference to an $O$ - $C$ diagram, to deduce the general form of period variation for GW Cep. It was found that the $O-C$ diagram could be interpreted as presenting two different forms of period change: an exclusively quasi-sinusoidal change with a period of 32.6 years and an eccentricity of 0.10 ; and a quasi-sinusoidal change with a period of 46.2 years and an eccentricity of 0.36 superposed on an upward parabola. Although a final conclusion is somewhat premature at present, the latter seems more plausible because late-type contact binaries allow an inter-exchange of both energy and mass between the component stars. The quasi-sinusoidal characteristics were interpreted in terms of a light-time effect due to an unseen tertiary component. The minimum masses of the tertiary component for both cases were calculated to be nearly the same as the 0.23-0.26 $M_{\odot}$-ranges which is hardly detectable in a light curve synthesis. The upward parabolic $O$ - $C$ diagram corresponding to a secular period increase of about $4.12 \times 10^{-8} \mathrm{~d} / \mathrm{yr}$ was interpreted as mass being transferred from the lesser to more massive component. The transfer rate for a conservative case was calculated to be about $2.66 \times 10^{-8} M_{\circ} / y r$ which is compatible with other W UMa-type contact binaries.

Keywords: eclipsing binaries, GW Cephei, period change, light-time effect, mass transfer

\section{INTRODUCTION}

Since the light variability of GW Cep (CSV 5941, BV 7, Sp = G3, P = 0.3188d) was discovered by Strohmeier (Geyer et al. 1955), it has been the subject of several investigations aiming to determine its basic system parameters. The first photoelectric light curve was measured by Meinunger \& Wenzel (1965), who classified GW Cep as a W UMa eclipsing binary with a G3 spectral type. After their study photoelectric or CCD observations of the system were made and/or analyzed by Hoffmann (1982), Kaluzny (1984), Landolt (1992), Pribulla et al. (2001a), and Lee et al. (2010). There have been no reports of spectro- scopic observations until now, with the detailed history of the system described by Lee et al. (2010). Through the results of the investigators above, the following consensus of the general photometric properties of GW Cep has been reached: (1) GW Cep belongs to Binnendijk's (1970) W-subtype of late-type contact binaries and has a total eclipse of about 24 minutes at primary eclipse; (2) two solar-type stars $\left(T_{1}=5,800 \mathrm{~K}\right.$ and $\left.T_{2}=6,108 \mathrm{~K}\right)$ with unequal mass $(q=0.379)$ and moderate contact $(f=0.174)$ are revolving circularly around their common center of mass, with a high orbital inclination of 84.4 degrees; (3) GW Cep has displayed remarkable light changes both during and between eclipses, implying strong magnetic activity in (c) This is an Open Access article distributed under the terms of the Creative Commons Attribution Non-Commercial License (http://creativecommons.org/licenses/by-nc/3.0/) which permits unrestricted non-commercial use, distribution, and reproduction in any medium, provided the original work is properly cited.
Received April 24, 2010 Accepted May 6, 2010

${ }^{\dagger}$ Corresponding Author

E-mail: kimch@chungbuk.ac.kr

Tel: +82-43-261-3139 Fax: +82-43-274-2312 
the system (Lee et al. 2010).

Period studies for the system have been carried out by Ragazzoni \& Barbieri $(1994,1996)$, Pribulla et al. (2001a), Qian (2003), Chochol et al. (2006), and Lee et al. (2010). A secular decrease of period was first considered by Ragazzoni \& Barbieri (1996) and later by Pribulla et al. (2001a), and Qian (2003). Chochol et al. (2006) suggested the possibility that the period of GW Cep may vary with either a single light-time effect (hereafter LITE) with a period of 32.6 years due to a tertiary body or a LITE with a short period of 13.5 years superposed on a downward parabola. Lee et al. (2010) showed that a single LITE with a period of 32.6 years would suffice to explain the period change of GW Cep with the latest measured and collected times of minima. The aim of this paper is to resolve the confusion regarding the period variation of the GW Cep system with new eclipse timing observations.

\section{OBSERVATIONS AND DATA REDUCTION}

BVR CCD observations of GW Cep were made on 15 nights from November through December 2008 with a 1-m reflector at the Jincheon station of the Chungbuk National University Observatory in Korea. An electrically cooled FLI 2K CCD imaging system with a 21.5' $\times 21.5^{\prime}$ field of view, and a standard $B V R$ filter set were used. GSC 4502-0538 and GSC 4502-0542 were chosen as the comparison and check stars, respectively. Our comparison star was the check star used by Lee et al. (2010). The camera exposure time ranged between $40 \mathrm{~s}$ and $200 \mathrm{~s}$ according to nighttime visilbility.

The instrumentation and reduction method used for the raw CCD frames have been described in detail by Jeong et al. (2009). The resultant standard errors of our observations in terms of comparison minus check star were about $\pm 0 .{ }^{\mathrm{m}} 003$ in blue, $\pm 0 .{ }^{\mathrm{m}} 009$ in yellow and $\pm 0 .{ }^{\mathrm{m}} 021$ in red, respectively. A total of 1,381 individual observations were obtained in three colors (469 in blue, 465 in yellow and 447 in red). New light curves are under analysis and will be published elsewhere. From our $B V R$ observations 19 new times of minimum lights were determined by the conventional Kwee \& van Woerden (1956) method. Each of these timings, as listed in Table 1, was a weighted-mean of three $B V R$ timings defining the same epoch.

\section{PERIOD STUDY}

For our study of the period variation of GW Cep, a total of 164 (46 visual, 4 sky patrol, 2 photographic, 16 photoelectric, and 95 CCD) times of minimum light were collected from a modern data-base (Kreiner et al. 2001) and from the recent literature. Table 1 lists all of the collected photoelectric and CCD minima. The minima marked by an asterisk in column 9 were determined with the consideration of two-spots model using the Wilson-Devinney binary code by Lee et al. (2010).

To see a general pattern of period change of GW Cep, the $(O-C)$ residuals of all 164 timings were calculated with Lee et al.'s (2010) linear ephemeris:

$$
C=\text { HJD } 2451799.49465+0 .{ }^{\mathrm{d}} 318831533 E .
$$

The $O-C$ diagram is shown in Fig. 1 where the dashed and solid lines represent the theoretical LITEs calculated with the orbital parameters (see the second column of Table 2) of Lee et al. (2010) and ours to be discussed later, respectively. Assorted symbols distinguish the timings according to observation method and type of eclipse. As seen in Fig. 1, it is clear that the period of GW Cep has varied in a cyclic way over time-interval of about 45 years, confirming Lee et al.'s (2010) result. We can undoubtedly rule out the possibility of any secular period decreases, which some previous investigators have proposed.

The cyclic behavior of the residuals, as seen in Fig. 1, has usually been interpreted in two mechanisms: a LITE due to an unseen third body; and a cyclic magnetic effect(s) in one or both of the components, as proposed

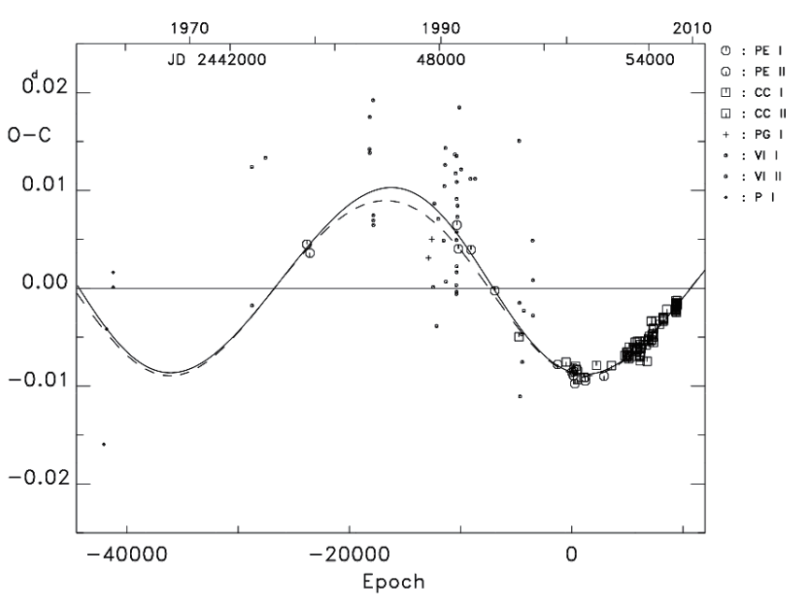

Fig. 1. History of the timings of minimum light for GW Cep against Eq (1). The residuals are coded by observational method. The dashed curve represents the sinusoidal term from the LITE ephemeris of Lee et al. (2010). The solid line represents our LITE orbit. 
Table 1. Photoelectric and CCD times of minimum lights of GW Cep.

\begin{tabular}{|c|c|c|c|c|c|c|c|c|}
\hline $\begin{array}{l}\text { Timing } \\
\text { (HJD2400000+) }\end{array}$ & Error & Min & Me & Epoch & $O-C_{1}$ & $O-C_{\text {ffull }}$ & $O-C_{2 \text { full }}$ & Reference \\
\hline 44200.48206 & 0.00004 & I & PE & -23834.0 & 0.003676 & 0.00036 & 0.00028 & Hoffmann (1982)* \\
\hline 44289.27556 & 0.00015 & II & PE & -23555.5 & 0.002777 & -0.00092 & -0.00083 & Hoffmann (1982)* \\
\hline 48504.3821 & 0.0010 & I & PE & -10335.0 & 0.005694 & 0.00087 & 0.00085 & Hubscher et al. (1992) \\
\hline 48544.87119 & 0.00008 & I & PE & -10208.0 & 0.003263 & -0.00137 & -0.00141 & Landolt (1992)* \\
\hline 48909.2948 & 0.0009 & I & PE & -9065.0 & 0.003179 & 0.00040 & 0.00025 & Hubscher et al. (1993) \\
\hline 49592.5452 & 0.0004 & I & PE & -6922.0 & -0.000992 & 0.00001 & -0.00002 & Agerer \& Hubscher (1995) \\
\hline 50283.447 & 0.002 & I & CCD & -4755.0 & -0.005705 & -0.00106 & -0.00087 & Diethelm (1996) \\
\hline 51391.5409 & 0.0002 & II & PE & -1279.5 & 0.008522 & 0.00012 & 0.00022 & Agerer \& Hubscher (2001) \\
\hline 51634.8091 & 0.0001 & II & CCD & -516.5 & -0.008282 & 0.00082 & 0.00086 & Nelson (2001) \\
\hline 51799.48420 & 0.00016 & I & $\mathrm{PE}$ & .0 & 0.009330 & -0.00002 & -0.00001 & Pribulla et al. (2001a)* \\
\hline 51845.39552 & 0.00011 & I & PE & 144.0 & 0.009656 & -0.00030 & -0.00030 & Pribulla et al. (2001a)* \\
\hline 51854.32356 & 0.00012 & I & $\mathrm{PE}$ & 172.0 & 0.008881 & 0.00048 & 0.00048 & Pribulla et al. (2001a)* \\
\hline 51858.30860 & 0.00010 & II & PE & 184.5 & 0.009227 & 0.00014 & 0.00014 & Pribulla et al. (2001a)* \\
\hline 51884.61089 & 0.00012 & I & PE & 267.0 & 0.010484 & -0.00110 & -0.00110 & Pribulla et al. (2001a)* \\
\hline 51900.3948 & 0.0002 & II & CCD & 316.5 & 0.008703 & 0.00070 & 0.00069 & Agerer \& Hubscher (2002) \\
\hline 51935.7847 & 0.0001 & II & CCD & 427.5 & 0.009030 & 0.00040 & 0.00038 & Nelson (2002) \\
\hline 51957.7830 & 0.0001 & II & CCD & 496.5 & 0.010061 & -0.00062 & -0.00064 & Nelson (2002) \\
\hline 51968.3054 & 0.0001 & II & PE & 529.5 & 0.009080 & 0.00037 & 0.00035 & Pribulla et al. (2001b) \\
\hline 52143.5022 & 0.0002 & I & CCD & 1079.0 & -0.009847 & -0.00034 & -0.00039 & Agerer \& Hubscher (2002) \\
\hline 52185.2687 & 0.0002 & I & $\mathrm{PE}$ & 1210.0 & 0.010192 & -0.00068 & -0.00073 & Pribulla et al. (2002) \\
\hline 52185.4285 & 0.0001 & II & PE & 1210.5 & 0.009808 & -0.00029 & -0.00035 & Pribulla et al. (2002) \\
\hline 52502.5070 & 0.0003 & I & CCD & 2205.0 & -0.008616 & 0.00077 & 0.00069 & Agerer \& Hubscher (2003) \\
\hline 52723.2963 & 0.0002 & II & $\mathrm{PE}$ & 2897.5 & 0.009699 & -0.00055 & -0.00064 & Pribulla et al. (2005) \\
\hline 52935.3199 & 0.0001 & II & CCD & 3562.5 & -0.008633 & 0.00017 & 0.00010 & Krajci (2005) \\
\hline 53322.2222 & 0.0001 & I & CCD & 4776.0 & -0.007603 & 0.00034 & 0.00030 & Kim et al. (2006) \\
\hline 53363.6701 & 0.0002 & I & CCD & 4906.0 & -0.007717 & 0.00011 & 0.00009 & Krajci (2006) \\
\hline 53378.0179 & 0.0001 & I & CCD & 4951.0 & -0.007307 & 0.00049 & 0.00046 & Kim et al. (2006) \\
\hline 53378.1769 & 0.0001 & II & CCD & 4951.5 & -0.007722 & 0.00007 & 0.00004 & Kim et al. (2006) \\
\hline 53378.3368 & 0.0001 & I & CCD & 4952.0 & -0.007238 & 0.00055 & 0.00053 & Kim et al. (2006) \\
\hline 53380.2497 & 0.0002 & I & CCD & 4958.0 & -0.007323 & 0.00046 & 0.00044 & Kim et al. (2006) \\
\hline 53385.9887 & 0.0005 & I & CCD & 4976.0 & -0.007279 & 0.00049 & 0.00047 & Kim et al. (2006) \\
\hline 53407.9878 & 0.0007 & I & CCD & 5045.0 & -0.007510 & 0.00020 & 0.00018 & Kim et al. (2006) \\
\hline 53433.6533 & 0.0001 & II & CCD & 5125.5 & -0.007895 & -0.00026 & -0.00028 & Chochol et al. (2006) \\
\hline 53449.4366 & 0.0002 & I & CCD & 5175.0 & -0.006724 & 0.00087 & 0.00085 & Chochol et al. (2006) \\
\hline 53449.5952 & 0.0001 & II & CCD & 5175.5 & -0.007539 & 0.00005 & 0.00004 & Chochol et al. (2006) \\
\hline 53491.5213 & 0.0001 & I & CCD & 5307.0 & -0.007700 & -0.00023 & -0.00024 & Pribulla et al. (2005) \\
\hline 53607.5766 & 0.0001 & I & CCD & 5671.0 & -0.006839 & 0.00028 & 0.00028 & Brat et al. (2007) \\
\hline 53614.5915 & 0.0002 & I & CCD & 5693.0 & -0.006218 & 0.00088 & 0.00088 & Brat et al. (2007) \\
\hline 53619.0544 & 0.0001 & I & CCD & 5707.0 & -0.006951 & 0.00013 & 0.00014 & Kim et al. (2006) \\
\hline 53663.05284 & 0.00009 & I & CCD & 5845.0 & -0.007172 & -0.00023 & -0.00022 & Lee et al. $(2010)^{*}$ \\
\hline 53664.00946 & 0.00019 & I & CCD & 5848.0 & -0.007045 & -0.00010 & -0.00009 & Lee et al. $(2010)^{*}$ \\
\hline 53664.16890 & 0.00015 & II & CCD & 5848.5 & -0.007020 & -0.00008 & -0.00007 & Lee et al. $(2010)^{*}$ \\
\hline 53665.92260 & 0.00010 & I & CCD & 5854.0 & -0.006890 & 0.00005 & 0.00006 & Lee et al. $(2010)^{*}$ \\
\hline 53666.08188 & 0.00006 & II & CCD & 5854.5 & -0.007025 & -0.00009 & -0.00008 & Lee et al. $(2010)^{*}$ \\
\hline 53666.24138 & 0.00006 & I & CCD & 5855.0 & -0.006941 & -0.00001 & 0.00000 & Lee et al. (2010)* \\
\hline 53672.6182 & 0.0021 & I & CCD & 5875.0 & -0.006738 & 0.00018 & 0.00019 & Brat et al. (2007) \\
\hline 53674.6912 & 0.0002 & II & CCD & 5881.5 & -0.006139 & 0.00077 & 0.00078 & Brat et al. (2007) \\
\hline 53730.4866 & 0.0033 & II & CCD & 6056.5 & -0.006143 & 0.00058 & 0.00060 & Brat et al. (2007) \\
\hline 53747.2237 & 0.0003 & I & CCD & 6109.0 & -0.007664 & -0.00099 & -0.00098 & Parimucha et al. (2007) \\
\hline 53763.4836 & 0.0001 & I & CCD & 6160.0 & -0.008138 & -0.00152 & -0.00150 & Parimucha et al. (2007) \\
\hline 53763.6436 & 0.0002 & II & CCD & 6160.5 & -0.007554 & -0.00094 & -0.00092 & Parimucha et al. (2007) \\
\hline 53764.2813 & 0.0002 & II & CCD & 6162.5 & -0.007516 & -0.00090 & -0.00088 & Parimucha et al. (2007) \\
\hline 53764.4405 & 0.0002 & I & CCD & 6163.0 & -0.007731 & -0.00112 & -0.00110 & Parimucha et al. (2007) \\
\hline 53765.3975 & 0.0003 & I & CCD & 6166.0 & -0.007224 & -0.00061 & -0.00059 & Parimucha et al. (2007) \\
\hline 53765.5577 & 0.0008 & II & CCD & 6166.5 & -0.006439 & 0.00017 & 0.00019 & Parimucha et al. (2007) \\
\hline 53777.99175 & 0.00010 & II & $\mathrm{CCD}$ & 6205.5 & -0.006793 & -0.00023 & -0.00020 & Lee et al. $(2010)^{*}$ \\
\hline
\end{tabular}


Table 1. (Continued)

\begin{tabular}{|c|c|c|c|c|c|c|c|c|}
\hline $\begin{array}{l}\text { Timing } \\
\text { (HJD2400000+) }\end{array}$ & Error & Min & $\mathrm{Me}$ & Epoch & $O-C_{1}$ & $O-C_{\text {lfull }}$ & $O-C_{2 \text { full }}$ & Reference \\
\hline 3989.05873 & 0.00010 & II & CCD & 6867.5 & 0.005855 & -0.00003 & 0.00001 & Lee et al. $(2010)^{*}$ \\
\hline 53992.08753 & 0.00007 & I & CCD & 6877.0 & -0.005948 & -0.00014 & -0.00010 & Lee et al. $(2010)^{*}$ \\
\hline 53992.24680 & 0.00011 & II & CCD & 6877.5 & -0.006093 & -0.00028 & -0.00024 & Lee et al. $(2010)^{*}$ \\
\hline 54026.99984 & 0.00023 & II & CCD & 6986.5 & -0.005619 & 0.00006 & 0.00010 & Lee et al. $(2010)^{*}$ \\
\hline 54067.6504 & 0.0002 & I & CCD & 7114.0 & -0.005996 & -0.00047 & -0.00042 & Brat et al. (2007) \\
\hline 54080.2461 & 0.0004 & II & CCD & 7153.5 & -0.004116 & 0.00137 & 0.00141 & Hubscher \& Walter (2007) \\
\hline 54080.4036 & 0.0003 & I & CCD & 7154.0 & -0.006031 & -0.00055 & -0.00051 & Hubscher \& Walter (2007) \\
\hline 54080.5650 & 0.0014 & II & CCD & 7154.5 & -0.004047 & 0.00143 & 0.00148 & Hubscher \&Walter (2007) \\
\hline 54097.3022 & 0.0007 & I & CCD & 7207.0 & -0.005468 & -0.00005 & -0.00001 & Diethelm (2007) \\
\hline 54129.98218 & 0.00008 & II & CCD & 7309.5 & -0.005653 & -0.00036 & -0.00031 & Lee et al. $(2010)^{*}$ \\
\hline 54130.14190 & 0.00007 & I & CCD & 7310.0 & -0.005348 & -0.00006 & -0.00001 & Lee et al. $(2010)^{*}$ \\
\hline 54134.12692 & 0.00013 & II & CCD & 7322.5 & -0.005714 & -0.00044 & -0.00039 & Lee et al. (2010)* \\
\hline 54137.6350 & 0.0002 & II & CCD & 7333.5 & -0.004774 & 0.00049 & 0.00053 & Krajci (2007) \\
\hline 54137.95237 & 0.00010 & II & CCD & 7334.5 & -0.006235 & -0.00097 & -0.00093 & Lee et al. (2010)* \\
\hline 54138.5914 & 0.0002 & II & CCD & 7336.5 & -0.004866 & 0.00039 & 0.00044 & Krajci (2007) \\
\hline 54211.2857 & 0.0002 & II & CCD & 7564.5 & -0.004007 & 0.00097 & 0.00102 & Dogru et al. (2007) \\
\hline 54314.4271 & 0.0001 & I & CCD & 7888.0 & -0.004396 & 0.00017 & 0.00021 & Brat et al. (2007) \\
\hline 54421.07667 & 0.00014 & II & CCD & 8222.5 & -0.003754 & 0.00037 & 0.00041 & Lee et al. $(2010)^{*}$ \\
\hline 54421.23618 & 0.00013 & I & CCD & 8223.0 & -0.003660 & 0.00047 & 0.00050 & Lee et al. $(2010)^{*}$ \\
\hline 54422.35188 & 0.00013 & II & CCD & 8226.5 & -0.003868 & 0.00025 & 0.00029 & Lee et al. $(2010)^{*}$ \\
\hline 54422.98953 & 0.00009 & II & CCD & 8228.5 & -0.003880 & 0.00024 & 0.00028 & Lee et al. $(2010)^{*}$ \\
\hline 54423.14906 & 0.00012 & I & CCD & 8229.0 & -0.003765 & 0.00035 & 0.00039 & Lee et al. (2010)* \\
\hline 54423.30818 & 0.00016 & II & CCD & 8229.5 & -0.004061 & 0.00006 & 0.00009 & Lee et al. (2010)* \\
\hline 54521.5093 & 0.0001 & II & CCD & 8537.5 & -0.002851 & 0.00086 & 0.00088 & Brat et al. (2008) \\
\hline 54774.98043 & 0.00023 & II & CCD & 9332.5 & -0.002269 & 0.00035 & 0.00031 & This paper \\
\hline 54775.13928 & 0.00011 & I & CCD & 9333.0 & -0.002834 & -0.00022 & -0.00025 & This paper \\
\hline 54776.09555 & 0.00015 & I & CCD & 9336.0 & -0.003057 & -0.00045 & -0.00048 & This paper \\
\hline 54776.09595 & 0.00009 & I & CCD & 9336.0 & -0.002657 & -0.00005 & -0.00008 & Lee et al. $(2010)^{*}$ \\
\hline 54780.24102 & 0.00010 & I & CCD & 9349.0 & -0.002388 & 0.00020 & 0.00017 & Lee et al. (2010)* \\
\hline 54781.03754 & 0.00016 & II & CCD & 9351.5 & -0.002946 & -0.00036 & -0.00039 & This paper \\
\hline 54781.19716 & 0.00022 & I & CCD & 9352.0 & -0.002741 & -0.00015 & -0.00019 & This paper \\
\hline 54781.19767 & 0.00004 & I & CCD & 9352.0 & -0.002231 & 0.00036 & 0.00032 & Lee et al. (2010)* \\
\hline 54781.35673 & 0.00007 & II & CCD & 9352.5 & -0.002586 & 0.00000 & -0.00004 & Lee et al. (2010)* \\
\hline 54782.15358 & 0.00015 & I & CCD & 9355.0 & -0.002814 & -0.00023 & -0.00027 & This paper \\
\hline 54782.95149 & 0.00019 & II & CCD & 9357.5 & -0.001981 & 0.00060 & 0.00056 & This paper \\
\hline 54783.11000 & 0.00016 & I & CCD & 9358.0 & -0.002886 & -0.00031 & -0.00034 & This paper \\
\hline 54787.09572 & 0.00015 & II & CCD & 9370.5 & -0.002552 & 0.00001 & -0.00003 & This paper \\
\hline 54788.21156 & 0.00009 & I & CCD & 9374.0 & -0.002620 & -0.00006 & -0.00010 & This paper \\
\hline 54789.16824 & 0.00006 & I & CCD & 9377.0 & -0.002433 & 0.00012 & 0.00008 & Lee et al. (2010)* \\
\hline 54789.32746 & 0.00013 & II & CCD & 9377.5 & -0.002628 & -0.00008 & -0.00012 & Lee et al. (2010)* \\
\hline 54789.96515 & 0.00005 & II & CCD & 9379.5 & -0.002600 & -0.00005 & -0.00009 & Lee et al. $(2010)^{*}$ \\
\hline 54790.12469 & 0.00007 & I & CCD & 9380.0 & -0.002476 & 0.00007 & 0.00003 & Lee et al. (2010)* \\
\hline 54790.12428 & 0.00019 & I & CCD & 9380.0 & -0.002886 & -0.00034 & -0.00038 & This paper \\
\hline 54795.06680 & 0.00016 & II & CCD & 9395.5 & -0.002244 & 0.00028 & 0.00024 & This paper \\
\hline 54796.02238 & 0.00023 & II & CCD & 9398.5 & -0.003157 & -0.00063 & -0.00068 & This paper \\
\hline 54800.00813 & 0.00008 & I & CCD & 9411.0 & -0.002793 & -0.00029 & -0.00033 & This paper \\
\hline 54800.16818 & 0.00012 & II & CCD & 9411.5 & -0.002158 & 0.00035 & 0.00030 & This paper \\
\hline 54800.96500 & 0.00013 & I & CCD & 9414.0 & -0.002415 & 0.00009 & 0.00004 & This paper \\
\hline 54811.96459 & 0.00032 & II & CCD & 9448.5 & -0.002491 & -0.00004 & -0.00009 & This paper \\
\hline 54818.97941 & 0.00044 & II & CCD & 9470.5 & -0.001950 & 0.00047 & 0.00042 & This paper \\
\hline 54826.94980 & 0.00015 & II & CCD & 9495.5 & -0.002332 & 0.00005 & 0.00000 & This paper \\
\hline
\end{tabular}

*The minima were determined with the consideration of two-spots model using the Wilson-Devinney binary code by Lee et al. (2010). 
by Applegate (1992) and later modified by Lanza et al. (1998). Lee et al. (2010) preferred the former because they failed to find any connection between the light variation and period change of GW Cep, which would be expected by the Applegate mechanism. No matter which of the two mechanisms is responsible for the seemingly cyclic oscillation in the observed $O-C$ residuals of GW Cep, Lee et al. (2010) thought that its Keplerian period has been constant for about $45 \mathrm{yr}$. Their survey, from the Atlas of $O-C$ Diagrams of Eclipsing Binary Stars (Kreiner et al., 2001) showed that only 6 systems (AT Aqr, GW Cep, V906 Cyg, V743 Sgr, RZ UMi and BP Vel) among a total sample of 65 contact binaries were found to have constant periods. Finally, they suggested that the six systems signify brief episodes of constant period behavior during thermal relaxation oscillation processes. Here we note that the time-interval of about $45 \mathrm{yr}$ covered by the observations corresponds to only about 1.4 times the 32.6 -yr oscillation cycle. In such a case, any secular changes of period by evolutionary effects, such as mass-transfer or massloss, could be suppressed by the dominant cyclic change of the period. Similar cases were found in their period study histories for contact binaries such as YY Eri (Kim 1992, Maceroni \& van't Veer 1994, Kim et al. 1997) and SS Ari (Kurpinska-Winiarska \& Zakrzewski 1990, Demircan \& Selam 1993, Kim et al. 2003). We thus investigated whether GW Cep fits this case.

Before our subsequent analysis of the times of minimum light, most of the timings were weighted according to the inversely-squared values of their published internal errors. As seen in Table 1, all photoelectric and CCD minima have errors. Among the previous 52 non-photoelectric and non-CCD timings not listed in Table 1, there were 29 minima without errors. These minima were as- signed an inversely-squared weight of their standard deviation of $\pm 0.0068 \mathrm{~d}$, reasonably given by Lee et al. (2010).

With the weight system above, all times of minima were separately fitted to a LITE ephemeris with and without a quadratic term, respectively, as follows:

and

$$
C_{1}=T_{\mathrm{o}}+P E+\tau
$$

$$
C_{2}=T_{\mathrm{o}}+P E+A E^{2}+\tau
$$

where $\tau$ is the light-time term with a parametric form taken from Irwin $(1952,1959)$. The Levenberg-Marquardt method (Press et al. 1992) was used to solve the parameters of Equations (2) and (3). The solution converged quickly, and the results are listed in Table 2 together with those of Lee et al. (2010), wherein the parenthesized values give the standard errors of the tabulated quantities. The $\sigma$ values in the ninth row of Table 2 denote the weighted standard deviations of residuals from all terms in Equations (2) and (3). These values fit Equation (3) slightly better than Equation (2). The solid line in Figure 1 was drawn using our single LITE orbital elements in the third column of Table 2. The parameters of Lee et al. (2010) were slightly modified with the inclusion of our latest timings. The $\left(O-C_{1}\right)$ and $\left(O-C_{\text {lfull }}\right)$ residuals calculated with the linear term and the full terms of Equation (3) are listed in the sixth and seventh columns of Table 1, respectively. The LITE elements derived with Equation (3) in the fourth column of Table 2, however, are quite different from both Lee et al.'s (2010) and our single LITE, especially the larger eccentricity of 0.36 and the longer period of $46.2 \mathrm{yr}$. The residuals from the linear term of Equation (3) were drawn in Figure 2 using the parabolic and cyclical components fitting to $\left(O-C_{2}\right)$ residuals. The

\begin{tabular}{|c|c|c|c|c|}
\hline \multirow{2}{*}{ Parameter } & \multirow{2}{*}{\begin{tabular}{|l|} 
Lee et al. (2010) \\
Third-body only \\
\end{tabular}} & \multicolumn{2}{|c|}{ This paper } & \multirow{2}{*}{ Unit } \\
\hline & & Third-body only & Third-body plus quadratic & \\
\hline$T_{\mathrm{o}}$ & $2451799.49280(26)$ & $2451799.49352(24)$ & $2451799.49465(23)$ & HJD \\
\hline$P$ & $0.318830882(32)$ & $0.318830878(28)$ & $0.318831533(22)$ & $\mathrm{d}$ \\
\hline K & $0.00896(42)$ & $0.00951(32)$ & $0.00951(32)$ & $\mathrm{d}$ \\
\hline$e$ & $0.076(40)$ & $0.104(44)$ & $0.361(35)$ & - \\
\hline$\omega$ & $221.8(2.1)$ & $181.5(2.8)$ & $164.1(3.1)$ & $\operatorname{deg}$ \\
\hline$T$ & $2450716(72)$ & 2449458(52) & 2449298(50) & HJD \\
\hline$P_{3}$ & $32.63(65)$ & $32.58(58)$ & $46.24(55)$ & $\mathrm{yr}$ \\
\hline$A$ & - & - & $1.80(45) \times 10^{-11}$ & $\mathrm{~d} / \mathrm{P}$ \\
\hline$\sigma$ & - & 0.00169 & 0.00166 & d \\
\hline$f\left(M_{3} / M \odot\right)$ & $0.00353(18)$ & $0.00427(16)$ & $0.00524(14)$ & $\mathrm{M}$. \\
\hline$M_{3}\left(i_{3}=90^{\circ}\right)$ & 0.21 & 0.23 & 0.26 & $\mathrm{M} \odot$ \\
\hline$M_{3}\left(i_{3}=60^{\circ}\right)$ & 0.25 & 0.27 & 0.30 & $\mathrm{M} \odot$ \\
\hline$M_{3}\left(i_{3}=30^{\circ}\right)$ & 0.47 & 0.51 & 0.58 & $\mathrm{M} \odot$ \\
\hline$d M / d t$ & - & - & $2.66 \times 10^{-8}$ & $\mathrm{M} \odot / \mathrm{yr}$ \\
\hline
\end{tabular}

Table 2. The derived LITE and secular ephemerides for GW Cep. 


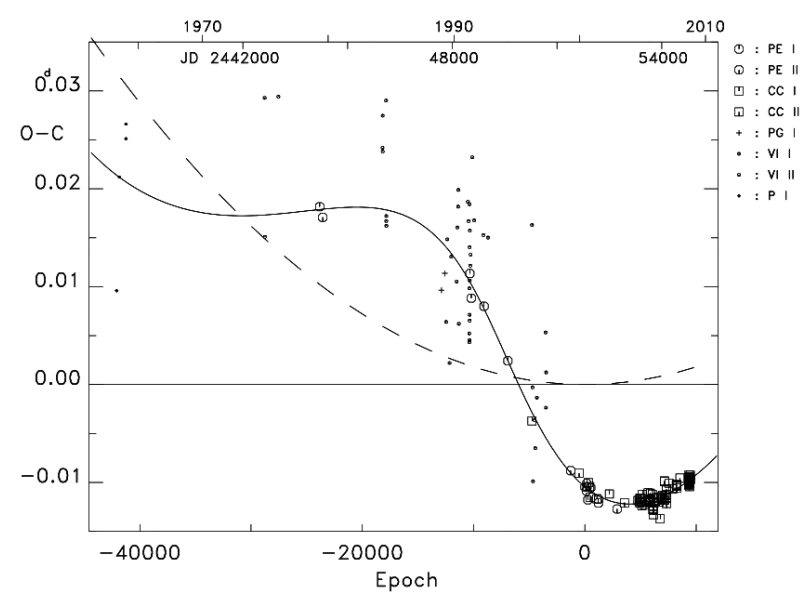

Fig. 2. The $\left(O-C_{2}\right)$ residuals of timings for GW Cep against the linear term of Eq. (3). The solid and dashed curves show non-linear terms and only the quadratic term of Eq. (3), respectively.

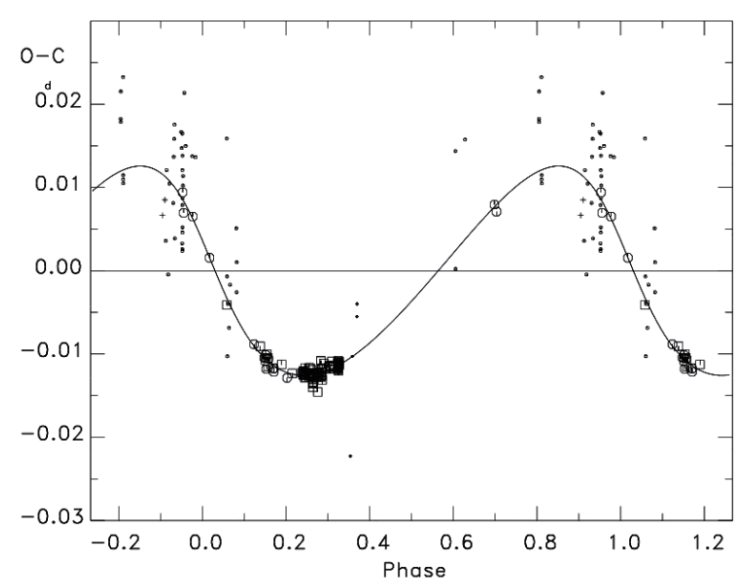

Fig. 3. The $\left(O-C_{2}\right)$ residuals phased with $P=46.2 \mathrm{yr}$ and $e=0.36$ from the solution in Table 2. The solid curve represents the projected LITE orbit of the barycenter of GW Cep caused by a third star.

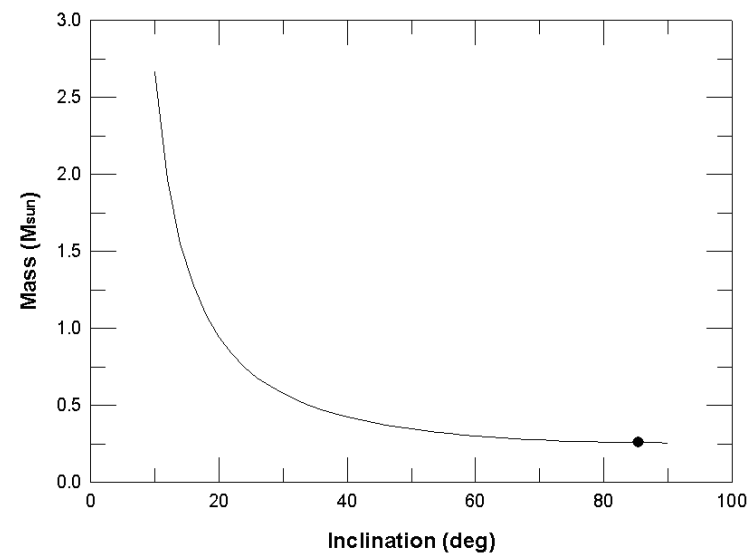

Fig. 4. A diagram of mass versus inclination of the tertiary star from the mass function in Table 2 . The large dot denotes the coplanar case between the eclipsing pair and the tertiary star.
$\left(O-C_{2 f u l l}\right)$ residuals from all terms are listed in the eighth column of Table 1. Figure 3 shows the $O$ - $C$ residuals from the linear and parabolic terms and the theoretical LITE curve phased with the third-body ephemeris of Table 2.

The 46.2 yr cyclic component of the period variability that appears to be present in the data set could be explained by either a LITE or an Applegate model, as discussed above. The latter interpretation is a possible mechanism explaining the apparent cyclic period change, because the two late-type stars in GW Cep could have strong magnetic activity and an activity cycle (Guinan \& Gimenez 1993) and strong light variations as intensively analyzed by Lee et al. (2010). However, because of insufficient light-curve data, it may not be possible to check whether the overall brightness of GW Cep has varied in the same way as the period change, which is what the Applegate model requires. In this case, the length of the activity cycle would be about $46 \mathrm{yr}$. The LITE interpretation for the cyclic component with an eccentric orbit $(e=0.36)$ gave a mass function $f\left(\mathrm{~m} / M_{\odot}\right)$ of 0.00524 for the LITE orbit. To estimate the mass of the third body, the mass function was solved as a function of the orbital inclination of the third body. A diagram of mass versus inclination in Fig. 4 shows a minimum mass of $0.26 M_{\circ}$ for the hypothetical tertiary body. At the moment, however, there are no other observations that support the thirdbody hypothesis. If the minimum mass of $0.26 M_{\odot}$ is adopted and the tertiary is assumed to be a main-sequence star, it would be a dM star with a low luminosity of 0.007 $L_{\circ}$ (see also Lee et al. 2010).

The parabolic component of the period change of GW Cep corresponds to a secular period increase of $+4.12 \times$ $10^{-8} \mathrm{~d} / \mathrm{yr}$ and implies a mass transfer of $2.66 \times 10^{-8} \mathrm{M}_{\circ} /$ yr from the less massive to the more massive star, that is, if the mass and angular momentum of the system are conserved and the masses for the eclipsing stars of $M_{\mathrm{h}}=$ $0.39 M_{\odot}$ and $M_{\mathrm{c}}=1.06 M_{\odot}$ given by Maceroni \& van't Veer (1996) are used. The mass transfer rate in the GW Cep system is moderate and is similar to the rates provided by YY Eri (Kim et al. 1997) and V432 Per (Lee et al. 2008).

\section{DISCUSSION AND CONCLUSIONS}

Nineteen new times of minima of GW Cep were determined from the $B V R$ CCD observations, which were carried out over fifteen nights in the winter season of 2008. A total of 164 timings available to us, including our own, were intensively analyzed to resolve the diverse and discordant interpretations proposed for the system's period 
change. At the early stage of period study, a secular decrease was proposed with timings then available (Pribulla et al. 2001, Qian 2003, Ragazzoni et al. 1996). Several years later, a 32-yr cyclic change of period was suggested with more accumulated minima (Chochol et al. 2006, Lee et al. 2010). Finally, this paper proposed a $46-y r$ periodicity superposed on an upward parabolic change of period. Our proposition was motivated by following factors: 1 ) the $32.6-y r$ periodicity completed only 1.4 cycles during the time-interval of about $45 \mathrm{yr}, 2$ ) in such a case, any secular changes of period by evolutionary effects, such as mass-transfer or mass-loss, could be suppressed by the dominant cyclic change of period, and 3) the latetype contact binaries are not free from mass transfer and energy exchange between components because they are not in thermal equilibrium (Lucy 1976, Stepien 2006). From our discussion above, we see that diverse historical interpretations of GW Cep result from the unpredictable behavior of its period variation, which may be ascribed to a natural process when finding the dynamical properties of the system. However, when the period behavior determined with timings within a short time-interval are really a part of oscillatory change with a longer period, any derived interpretations may have a high probability of being erroneous. Furthermore, the astrophysically important parameters obtained (e.g., mass-transfer rates and LITEs) could be wrongly used in understanding the evolutionary and/or dynamical states of contact binaries. For this reason, the suggestion of Rovithis-Livaniou et al. (2005) that "it is desirable to wait and see if it will be repeated for the case of any periodicity corresponding to the time-interval covered by observations" would be properly applicable to the historical period studies of the GW Cep system.

In conclusion, the apparent secondary variation of GW Cep's period varies in a sinusoidal way, superposed on the long-term upward parabolic variation. The secularly increasing rate of the period is deduced to be 0.36 s per century $\left(+4.12 \times 10^{-8} \mathrm{~d} / \mathrm{yr}\right)$, implying a mass transfer of $2.66 \times 10^{-8} \mathrm{M}_{\circ} / \mathrm{yr}$ from the less massive to the more massive star for the conservative case. The mass transfer may be a possible cause of the hot spot near the neck of the facing hemisphere of the cooler, more massive star, which was consistently found in the light curve synthesis by Lee et al. (2010). The period of the quasi-sinusoidal variation is about $46.2 \mathrm{yr}$. This period could arise from a LITE due to the gravitational effect of a third body or from a cyclic period modulation due to the magnetically active component stars. The tertiary body was deduced to be a low-mass $\left(m_{3}=0.26 M_{\odot} \sin i\right)$ and a low luminos- ity $\left(L_{3}=0.007 L_{\circ}\right) \mathrm{dM}$ companion moving in an eccentric $(e=0.36)$ orbit. However, there were no other observations supporting the tertiary body model. Although the historical light-curve variations indicate star-spots and possible high levels of magnetic dynamo activity, the possibility that the 46.2-yr periodicity arises from the varying magnetic activity of the stars seems unlikely, according to Lee et al.'s (2010) intensive investigation on that matter. At the same time, the dynamical picture of GW Cep is far from reality. The obvious way to try to further understand GW Cep is to obtain more data of all kinds to determine the following: magnetodynamic activity (e.g., cyclic effects in emission lines, maculation effects in the photometry, or radio or other EUVE and X-ray data); and other third-body effects (e.g., cyclic variations of gamma velocity, third-body spectrum in high resolution spectroscopy, detection of any third light in high precision photometry, or cyclic position-changes in astrometry).

\section{ACKNOWLEDGMENTS}

This work was supported by the research grant of the Chungbuk National University in 2008.

\section{REFERENCES}

Agerer, F. \& Hubscher, J. 1995, IBVS, 4222, 1

Agerer, F. \& Hubscher, J. 2001, IBVS, 5016, 1

Agerer, F. \& Hubscher, J. 2002, IBVS, 5296, 1

Agerer, F. \& Hubscher, J. 2003, IBVS, 5484, 1

Applegate, J. H. 1992, ApJ, 385, 621, doi: 10.1086/170967

Binnendijk, L. 1970, VA, 12, 217, doi: 10.1016/00836656(70)90041-3

Brat, L., Smelcer, L., Kueakova, H., Ehrenberger, R., Kocian, R., Lomoz, F., Urbaneok, L., Svoboda, P., Trnka, J., Marek, P., Doevìny, R., Uhlao, R., Poddany, S., Zasche, P., \& Skarka, M. 2008, OEJV, 94, 1

Brat, L., Zejda, M., \& Svoboda, P. 2007, OEJV, 74, 1

Chochol, D., Pribulla, T., Vanko, M., Mayer, P., Wolf, M., Niarchos, P., Gazeas, K., Manimanis, V., Brat, L., \& Zejda, M. 2006, Ap\&SS, 304, 93, doi: 10.1007/s10509-006-9160-6

Demircan, O. \& Selam, S. O. 1993, A\&A, 267, 107

Diethelm, R. 1996, BBSAG Bull, 112, 12

Diethelm, R. 2007, IBVS, 5781, 1

Dogru, S. S., Dogru, D., \& Donmez, A. 2007, IBVS, 5795, 1

Geyer, E., Kippenhann, R., \& Strohmeier, W. 1955, BamKV, 9, 1

Guinan, E. F. \& Gimenez, A. 1993, in Astrophysics and Space Science Library v 177: The Realm of Interacting Binary 
Stars, eds. J. Sahade, G. E. McCluskey, \& Y. Kondo (Dordrecht: Kluwer Academic Publishers), p.51

Hoffman, M. 1982, Ap\&SS, 83, 195, doi: 10.1007/BF00648552 Hubscher, J., Agerer, F., \&Wunder, E. 1992, BAV Mitt, 60, 1 Hubscher, J., Agerer, F., \& Wunder, E. 1993, BAV Mitt, 62, 1 Hubscher, J. \& Walter, F. 2007, IBVS, 5761, 1 Irwin, J. B. 1952, ApJ, 116, 211, doi: 10.1086/145604

Irwin, J. B. 1959, AJ, 64, 149, doi: 10.1086/107913

Jeong, J.-H., Kim, C.-H., Lee, Y. S., \& Yoon, J.-N. 2009, JASS, 26, 157

Kaluzny, J. 1984, AcA, 34, 217

Kim, C.-H. 1992, in Evolutionary Processes in Interacting Binary Stars Proceedings of the 151st Symposium of the International Astronomical Union, held in Cordoba, Argentina, August 5-9, 1991, eds. Y. Kondo, R. F. Sistero, \& R. S. Polidan (Boston, MA: Kluwer Academic Publishers), pp.383-386

Kim, C.-H., Jeong, J.-H., Demircan, O., Muyesseroglu, Z., \& Budding, E. 1997, AJ, 114, 2753, doi: 10.1086/118684

Kim, C.-H., Lee, C.-U., Yoon, Y.-N., Park, S.-S., Kim, D.-H., Cha, S.-M., \&Won, J.-H. 2006, IBVS, 5694, 1

Kim, C.-H., Lee, J. W., Kim, S. L., Han, W., \& Koch, R. H. 2003, AJ, 125, 322, doi: 10.1086/344808

Krajci, T. 2005, IBVS, 5592, 1

Krajci, T. 2006, IBVS, 5690, 1

Krajci, T. 2007, IBVS, 5806, 1

Kreiner, J. M., Kim, C.-H., \& Nha, I.-S. 2001, An Atlas of O-C Diagrams of Eclipsing Binary Stars. Pt. 1-6 (Krakow: Wydaw. Naukowe Akademii Pedagogicznej)

Kurpinska-Winiarska, M. \& Zakrzewski, B. 1990, IBVS, 3485, 1

Kwee, K. K. \& van Woerden, H. 1956, BAN, 12, 327

Landolt, A. U. 1992, PASP, 104, 336, doi: 10.1086/133002

Lanza, A. F., Rodono, M., \& Rosner, R. 1998, MNRAS, 296, 893, doi: 10.1046/j.1365-8711.1998.01446.x

Lee, J. W., Youn, J.-H., Han, W., Lee, C.-U., Kim, S.-L., Kim, H.-I., Park, J.-H., \& Koch, R. H. 2010, AJ, 139, 898, doi: $10.1088 / 0004-6256 / 139 / 3 / 898$
Lee, J. W., Youn, J.-H., Kim, C.-H., Lee, C.-U., \& Kim, H.-I. 2008, AJ, 135, 1523, doi: 10.1088/0004-6256/135/4/1523

Lucy, L. B. 1976, ApJ, 205, 208, doi: 10.1086/154265

Maceroni, C. \& van't Veer, F. 1994, A\&A, 289, 871

Maceroni, C. \& van't Veer, F. 1996, A\&A, 311, 523

Meinunger, L. \& Wenzel, W. 1965, Mitt Veranderliche Sterne, Sonnenberg, 2, 164

Nelson, R. H. 2001, IBVS, 5040, 1

Nelson, R. H. 2002, IBVS, 5224, 1

Parimucha, S., Vanko, M., Pribulla, T., Hambalek, L., Dubovsky, P., Baludansky, D., Petrik, K., Chrastina, M., \& Urbancok, L. 2007, IBVS, 5777, 1

Press, W. H., Teukolsky, S. A., Vetterling, W. T., \& Flannery, B. P. 1992, Numerical Recipes (Cambridge: Cambridge University Press), Ch.15

Pribulla, T., Baludansky, D., Chochol, D., Chrastina, M., Parimucha, S., Petrik, K., Szasz, G., Vanko, M., \& Zboril, M. 2005, IBVS, 5668, 1

Pribulla, T., Vanko, M., Chochol, D., \& Parimucha, S. 2001a, CoSka, 31, 26

Pribulla, T., Vanko, M., Parimucha, S., \& Chochol, D. 2001b, IBVS, 5056, 1

Pribulla, T., Vanko, M., Parimucha, S., \& Chochol, D. 2002, IBVS, 5341, 1

Qian, S. 2003, MNRAS, 342, 1260, doi: 10.1046/j.13658711.2003.06627.x

Ragazzoni, R. \& Barbieri, C. 1994, PASP, 106, 683, doi: $10.1086 / 133429$

Ragazzoni, R. \& Barbieri, C. 1996, IBVS, 4293, 1

Rovithis-Livaniou, H., Tsantilas, S., Kalimeris, A., Rovithis, P., Djurasevic, G., \& Voloshina, I. 2005, in The Light-Time Effect in Astrophysics, Proceedings of ASP Conference Series, Vol 335, held in Brussels 19-22 July 2004, ed. C. Sterken (San Francisco, CA: Astronomical Society of the Pacific), p.251

Stepien, K. 2006, AcA, 56, 199 